\title{
Characterizing the landscape of peritoneal exosomal microRNAs in patients with ovarian cancer by high-throughput sequencing
}

\author{
YUANKUN LI ${ }^{1}$, CUIHUA LIU ${ }^{2}$, YUMEI LIAO ${ }^{1}$, WULIANG WANG ${ }^{1}$, BIN HU $^{1}$, XIAOQIN LU $^{1}$ and JINQUAN CUI ${ }^{1}$ \\ ${ }^{1}$ Department of Gynecology, The Second Affiliated Hospital of Zhengzhou University; \\ ${ }^{2}$ Department of Gynecology, Zhengzhou First People's Hospital, Zhengzhou, Henan 450000, P.R. China
}

Received November 28, 2017; Accepted June 13, 2018

DOI: $10.3892 / \mathrm{ol} .2018 .9558$

\begin{abstract}
In the present study, differentially expressed microRNAs (miRNAs) in peritoneal exosomes that were isolated from 10 patients with epithelial ovarian cancer (EOC) with metastasis in the abdominal cavity and 10 participants without cancer (NC) were identified. These differentially expressed miRNAs that were revealed by next-generation sequencing were categorized by Gene Ontology enrichment and Kyoto Encyclopedia of Genes and Genomes pathway enrichment analysis of their target genes. Notably, two miRNAs that were associated with EOC-miR-149-3p and miR-222-5p-were identified. There were significant differences in expression of miR-149-3p and miR-222-5p between EOC and NC samples, and the effect of the expression level of the two miRNAs on the patient survival was identified using publicly available data from The Cancer Genome Atlas. There is an association between these two miRNAs and EOC, that was further verified by reverse transcription-quantitative polymerase chain reaction in peritoneal exosomes from 10 patients with EOC and NC participants. These results indicated that miR-149-3p and miR-222-5p might be novel biomarkers for evaluating the prognosis of patients with EOC and that these two miRNAs might have potential therapeutic values.
\end{abstract}

\section{Introduction}

Peritoneal metastasis is a key process during the development of chemo-resistance and postoperative relapse in ovarian cancer and leads to a poor prognosis and a high mortality rate $(1,2)$. Based on observations in clinical practice, $~ 80 \%$ of patients with ovarian cancer were diagnosed with peritoneal metastasis with a considerable proportion of the cases developed during early or medium stages of ovarian cancer progression (3).

Correspondence to: Professor Jinquan Cui, Department of Gynecology, The Second Affiliated Hospital of Zhengzhou University, 2 Jingba Road, Jinshui, Zhengzhou, Henan 450000, P.R. China E-mail: cuijinquan1952@yeah.net

Key words: epithelial ovarian cancer, next-generation sequencing, microRNA, miR-149-3p, miR-222-5p
Changes in gene expression, intracellular signal transduction and cell morphology during ovarian cancer cell metastasis are closely associated with the gain of stemness, maintenance and resistance to chemotherapy- or radiation-induced apoptosis, suggesting that these tumor biological characteristics are governed by number of conserved mechanisms (4-6).

Somatic copy number variation and change in the level of transcription are well-recognized key steps in the progression of ovarian cancer and other types of cancer (7-9). MicroRNAs (miRNAs/miRs) together with other non-coding RNAs comprise a sophisticated gene expression regulatory network. The disorder or alteration of the gene expression regulatory network often leads to the pathogenesis of different diseases, particularly those largely affected by changes in gene expression, including inflammatory disease and cancer (10-12). The alterations in the expression of different miRNAs have been linked to ovarian cancer progression in different aspects, such as proliferation, invasion and metastasis (13-15). However, current investigations are mainly in vitro experiments that are focused on individual miRNAs using cancer cell lines, yielding results unable to accurately reflect the effects in patients and hard to be translated into therapeutic strategy (13-15).

Another limitation of the majority of current studies focusing on miRNAs in ovarian cancer is that microRNAs not only deposit in cells but also in extracellular vesicles, including microvesicles and exosomes (16). Exosomes as important extracellular microenvironment components can be secreted from and received by different cells, transporting contents, including effector proteins and RNAs between cells, and thus mediating intercellular signal transduction (16). Focusing only on miRNAs in cancer cells may therefore miss important information. It was raised by several studies that exosomes may have an important role in regulating ovarian cancer metastasis and development (17-19).

The aim and scope of the present study was to identify miRNAs in exosome that may function as promoters of oncogenesis or peritoneal metastasis of ovarian cancer. By comparing the miRNA expression patterns in exosomes that were isolated from 10 samples of ascites from patients with epithelial ovarian cancer (EOC) and 10 non-cancerous (NC) peritoneal lavage samples. It was revealed that two exosomal miRNAs, miR-149-3p and miR-222-5p, were upregulated in ovarian cancer ascites-derived exosomes compared with the non-cancerous samples. It was further confirmed that the 
increased expression levels of these two miRNAs significantly correlated with the survival of patients with ovarian cancer, suggesting the prognostic value of the two miRNAs for malignant ovarian cancer.

\section{Materials and methods}

Participants and isolation of peritoneal exosomes. The present study was approved by the Medical Ethics Committee of the Second Affiliated Hospital of Zhengzhou University (Henan, China). A total of 10 patients with peritoneal metastatic epithelial ovarian cancer (EOC group) and 10 subjects without epithelial ovarian cancer with acute pelvic peritonitis (NC group) were enrolled in the present study. The clinical data of the participants are described in Table I. All participants were recruited at The Second Affiliated Hospital of Zhengzhou University (Zhengzhou, China), and written informed consent was obtained from each participant prior to enrolment. Peritoneal exosomes were isolated from a sample of ascites from each patient with EOC or a peritoneal lavage sample from subjects in the NC group using ExoQuick ${ }^{\mathrm{TM}}$ exosome precipitation solution (Invitrogen; Thermo Fisher Scientific, Inc., Waltham, MA, USA) following the manufacturer's instructions. Briefly, each sample of ascites or peritoneal lavage specimen was pre-cleared by centrifugation at $3,000 \mathrm{x} \mathrm{g}$ for $15 \mathrm{~min}$ under $4^{\circ} \mathrm{C}$ to remove cells or cell debris followed by mixing with precipitation solution on a 4:1 ratio $(\mathrm{v} / \mathrm{v})$. The mixture was then refrigerated under $4^{\circ} \mathrm{C}$ for $12 \mathrm{~h}$, after which the exosomes in the mixture were pelleted by centrifugation at $1,500 \mathrm{xg}$ for $30 \mathrm{~min}$ under $4^{\circ} \mathrm{C}$. The exosome pellet extracted from each sample was then re-suspended in $200 \mu$ l PBS, validated by a transmission electron microscopy and stored under $4^{\circ} \mathrm{C}$ for $\leq 1 \mathrm{~h}$ before further analysis. A total of $30 \mu \mathrm{l}$ supernatant from exosome isolation and $20 \mu \mathrm{l}$ of each exosome sample suspended in PBS were subjected to western blotting using a mouse monoclonal antibody against human CD63 (dilution of 1:1,000) (catalog no. ab213090; Abcam, Cambridge, UK) to evaluate the efficiency of exosome precipitation. In western blotting, the ProteoPrep ${ }^{\circledR}$ Total Extraction Sample kit (Sigma-Aldrich, Merck KGaA Darmstadt, Germany) was to separate proteins from exosomes according to the manufacturer's protocol. Samples were normalized for protein concentration by a BCA assay. The volumes $(20 \mu \mathrm{g})$ of each sample were loaded on a acrylamide-bisacrylamide (10 or $12.5 \%)$ gel. Following electrophoresis, the protein was transfected with PVDF membrane. Skimmed milk powder (5\%) blocked PVDF for $3 \mathrm{~h}$ at room temperature. The secondary antibody (Goat Anti-mouse IgG H\&L, catalog no. ab6785, Abcam, Cambridge, UK) has a dilution of 1:5,000, incubated in TBS-T buffer (Beijing Solarbio Science \& Technology Co., Ltd., Beijing, China) for $1 \mathrm{~h}$ at room temperature. Proteins were visualized using an enhanced chemiluminescence solution (Life Sciences; Thermo Fisher Scientific, Inc.).

High-throughput sequencing of peritoneal exosomal miRNAs. A total of 2 sequencing libraries of peritoneal exosomal miRNAs from EOC- or NC-group specimens were constructed using a sequencing library construction kit provided by Gene Denovo (Guangzhou, China) following manufacturer's instructions. Briefly, 10 samples of PBS-suspended exosomes
(80 $\mu$ l) from participants in each group were mixed, and total RNA was extracted from the mixture using TRIzol. Small RNAs with 18-30 nucleotides were separated by SDS-PAGE (15\%). After the ligation of $5^{\prime}$ and $3^{\prime}$ adapters to both ends of small RNAs by nested-polymerase chain reaction (PCR), the ligation products were reversely transcribed and amplified by PCR. The products (size, 140-160 bp) were further separated by agarose gel (3.5\%) to generate the cDNA library. A total of 2 cDNA libraries generated from EOC or NC samples were then subjected to high throughput sequencing by Gene Denovo using the Illumina HiSeqTM 2500 system (Illumina, Inc., San Diego, CA, USA).

Pre-processing of raw data and miRNA identification. To obtain small RNA clean reads, raw reads data were first filtered using the steps as follows: i) Removal of low-quality reads containing $>1$ low-quality (quality score $\leq 20$ ) base or containing unknown nucleotides $(\mathrm{N})$; ii) removal of reads without 3 ' or 5 ' adapters; iii) Removal of reads containing 3 ' and 5 ' adapters but no small RNA fragments; iv) removal of reads containing ploy-A sequence within small RNA fragments and v) removal of reads that are shorter than 18 nucleotides.

All clean reads were then aligned to human small RNA sequence data that were obtained from GeneBank (release 209.0, https://www.ncbi.nlm.nih.gov/genbank/) and Rfam databases (version 11.0, http://rfam.xfam.org/) to filter any ribosomal RNA, small cytoplasmic RNA, small nucleolar RNA, small nuclear RNA or transfer RNA. The clean reads were also aligned to a reference human genome [Homo sapiens (assembly GRCh38.p12)] to remove reads mapped to exons or introns that might be fragments from mRNA degradation or reads mapped to repeat sequences. Filtered small RNA clean reads were then referred to as clean tags, which were mapped in miRBase database (release 21, http://www.mirbase. org/index.shtml) to annotate any known human miRNA.

Analysis of miRNA expression. The expression level of every annotated miRNA in EOC or NC sample group was first normalized to transcripts per million (TPM) using the following formula:

$$
\mathrm{TPM}=\frac{\text { counts of miRNA }}{\text { Total counts of clean tags }} \times 10^{6}
$$

and $\mathrm{A} \log _{2}(\mathrm{FC})$ value of each annotated miRNA in NC or EOC sample group was calculated using the following formula:

$$
\log _{2}(\mathrm{FC})=\log _{2}\left(\frac{\mathrm{TPM}_{\mathrm{EOC}}}{\mathrm{TPM}_{\mathrm{NC}}}\right)
$$

where $\mathrm{TPM}_{\mathrm{EOC}}$ and $\mathrm{TPM}_{\mathrm{NC}}$ were TPM of a miRNA in EOC and $\mathrm{NC}$ samples, respectively, and all $\mathrm{TPM}=0$ were adjusted to TPM=0.01 to avoid infinity error. miRNAs with $\log _{2}(F C)>1$ $\mathrm{OR}<-1$ between EOC and NC samples were considered upregulated or downregulated, respectively. The annotated miRNAs were clustered according to their expression patterns to generate the heat map of miRNA expression in NC and EOC samples.

Gene set enrichment analysis of the target genes of miRNAs. Target gene candidates of annotated miRNAs in each sample 
Table I. Thermocycling conditions for quantitative polymerase reaction.

\begin{tabular}{|c|c|c|c|c|}
\hline Cycles & Steps & $\begin{array}{c}\text { Temperature } \\
\left({ }^{\circ} \mathrm{C}\right)\end{array}$ & Time & Detection \\
\hline 1 & $\begin{array}{l}\text { Initial } \\
\text { denaturation }\end{array}$ & 95 & $10 \mathrm{~min}$ & No \\
\hline \multirow[t]{3}{*}{40} & Denaturation & 95 & $10 \mathrm{sec}$ & No \\
\hline & Annealing & 75 & $20 \mathrm{sec}$ & No \\
\hline & Extension & 72 & $20 \mathrm{sec}$ & Yes \\
\hline
\end{tabular}

group were first predicted using RNAhybrid (version 2.1.2, https://bibiserv.cebitec.uni-bielefeld.de/rnahybrid/), miRanda (version. 3.3a, http://miranda.org.uk/) and TargetScan (version. 7.0, http://genes.mit.edu/targetscan.test/ucsc.html) databases. The genes predicted using all three databases were considered potential target genes. The preliminary miRNA pathway enrichment analysis was performed using the DIANA-miRPath (version 3, http://snf-515788.vm.okeanos. grnet.gr/) online software. DIANA-miRPath performs GO and KEGG enrichment analysis of genes in TarBase (version 7, http://diana.imis.athena-innovation.gr/DianaTools/index. php? $\mathrm{r}=$ tarbase/index) that have been validated as targets of given miRNAs. For Gene Ontology (GO) enrichment analysis, predicted target genes were mapped to GO terms in the Gene Ontology database (http://www.geneontology. org/). Significantly enriched GO terms were determined by an adjusted $\mathrm{P}<0.05$ that was calculated by hyper-geometric distribution and false discovery rate correction. For Kyoto Encyclopedia of Genes and Genomes (KEGG) pathway enrichment analysis, predicted miRNAs target genes were mapped to KEGG annotation in the KEGG database (http://www. genome.jp/kegg/pathway.html), and significantly enriched KEGG annotations were determined using the same method as that for Gene Ontology enrichment analysis.

Reverse transcription-quantitative polymerase chain reaction (RT-qPCR) of miR-149-3p and miR-222-5p in exosome samples. The expression levels of miR-149-3p or miR-222-5p in exosomes from 10 patients with epithelial ovarian cancer (EOC group) and 10 subjects without epithelial ovarian cancer (NC group) were evaluated by RT-qPCR using a custom-made microRNA qPCR kit (GeneCopoeia, Inc., Rockville, MD, USA) following the manufacturer's instructions. miRNAs were first reverse transcribed using a reverse transcription reaction (miRNA First-Strand cDNA Synthesis kit, GeneCopoeia, Inc., Rockville, MD, USA) mix at $37^{\circ} \mathrm{C}$ for $60 \mathrm{~min}$ followed by inactivation at $85^{\circ} \mathrm{C}$ for $5 \mathrm{~min}$. The primer sequence for has-miR-149-3p detection was 5'-GGCUCCGUGUCUUCA CUCCCAAA-3' and for has-miR-222-5p was 5'-AGUAGC CAGUGUAGAUCCUAAA-3'. qPCR reaction was performed following a standard three-step method as described in Table I.

The qPCR reaction was performed using a SimpliAmp thermocycler (Thermo Fisher Scientific, Inc.) and was monitored by a SYBR Green fluorescent dye. Semi-quantitative analysis was first performed to normalize the expression level of detected microRNAs to snRNA U6 (GeneCopoeia, Inc.) in each sample using the $2^{-\Delta \Delta C q}$ method. The fold change (FC) of microRNA expression level in each EOC-group sample was compared to the mean expression level in the $\mathrm{NC}$ group samples (20).

Kaplan-Meier curve analysis and statistical analysis. The associations between the expression of specific miRNAs and the survival of patients with ovarian cancer were investigated by Kaplan-Meier curve (with a log-rank test) that was generated using the UCSC Xena browser (http://xena.ucsc. edu/). The sequencing data from 595 publically available samples from patients with ovarian cancer and clinical record from The Cancer Genome Atlas ovarian cancer database (https://cancergenome.nih.gov/) were analyzed. Graphpad Prism software (version 7.0; GraphPad Software, Inc., La Jolla, CA, USA) was employed for statistical analysis unless otherwise indicated. Student's t-test was used for significance test, and $\mathrm{P}<0.05$ was considered statistically significant.

\section{Results}

Differentially expressed peritoneal exosomal miRNAs between participants without cancer and patients with epithelial ovarian cancer. To characterize the differences in peritoneal exosomal miRNA expression patterns between NC participants and patients with EOC, exosomes were isolated from 10 samples of ascites from patients with ovarian cancer and 10 peritoneal lavage samples from participants without cancer. The clinical information of these patients is shown in Table II. Following total exosome precipitation as verified by western blotting and transmission electron microscopy (Fig. 1), small RNAs from two groups of exosome samples were extracted, and differentially expressed miRNA between NC and EOC sample were detected by next-generation sequencing. Subsequently, bioinformatic analysis was performed. As described in the materials and method section, annotated miRNAs with $\mathrm{TPM}_{\mathrm{EOC}}$ two-fold higher compared with $\mathrm{TPM}_{\mathrm{NC}}$ with $\log _{2}(\mathrm{FC})>1$ were considered upregulated in EOC samples compared to NC samples. Similarly, miRNAs with $\log _{2}(\mathrm{FC})<-1$ were determined to be downregulated. Among the 748 annotated miRNAs, 249 miRNAs were upregulated and 317 were downregulated in EOC samples compared with NC samples (Fig. 2A and B). These differentially expressed miRNAs were clustered according to changes in expression level in a heat map (Fig. 2C) to further illustrate that there are different patterns of miRNA expression between $\mathrm{NC}$ and EOC samples. These data indicated that miRNAs in peritoneal exosomes were differentially expressed between NC participants and patients with EOC.

GO enrichment and KEGG pathway enrichment analysis of the target genes of differentially expressed miRNAs. miRNAs regulate the expression level of their specific target genes mostly by decreasing the efficiency of protein translation, therefore affecting different biological processes in cells. Considering that peritoneal exosomes are secretory extracellular vesicles from cells within the abdominal cavity and that exosomal contents may affect the biological processes in recipient cells when fused with exosome, changes in the expression of peritoneal exosomal miRNAs may therefore 
Table II. Clinical data of patients with epithelial ovarian cancer $(n=10)$ and subjects without epithelial ovarian cancer $(n=10)$.

\begin{tabular}{lcc}
\hline Variables & EOC & NC \\
\hline No. of participants & 10 & 10 \\
Age & & \\
$<48$ & 3 & 6 \\
$\geq 48$ & 7 & 4 \\
Pathology & & N/A \\
Benign & 0 & N/A \\
Borderline & 3 & N/A \\
Malignant & 7 & N/A \\
Histological grade & & N/A \\
Well-differentiated & 1 & N/A \\
Moderately differentiated & 7 & N/A \\
Poorly differentiated & 2 & N/A \\
FIGO stages & & N/A \\
I-II & 3 & N/A \\
III-VI & 7 & N/A \\
Volume of ascites & & N/A \\
Nonelsmall & & N/A \\
Moderate & 2 & N/A \\
Large & 3 & N/A \\
\hline
\end{tabular}

FIGO, International Federation of Gynecology and Obstetrics (Fédération Internationale de Gynécologie et d'Obstétrique).

reflect or cause physiopathological changes in peritoneal cells and the associated microenvironment.

To investigate the potential effect of changes in the expression pattern of peritoneal exosomal miRNAs on EOC oncogenesis or abdominal cavity metastasis, GO enrichment and KEGG pathway enrichment analysis were performed on the predicted target genes of upregulated and downregulated miRNAs. A total of 44,955 genes were identified as target genes of all differentially expressed miRNAs. The significantly enriched GO terms of the genes targeted by upregulated and downregulated miRNAs are summarized in Tables III and IV, respectively, and further compared in Fig. 3. Notably, the genes targeted by upregulated miRNAs encrypt proteins that are mostly located on the cell membrane or in the extracellular microenvironment (EM) that is involved in signaling and cell communication, implying that the upregulation of these miRNAs might inhibit cell-to-cell or cell-to-EM communication. To get a better understanding of the effect of the change in the expression levels of peritoneal exosomal miRNAs between $\mathrm{NC}$ and EOC samples, KEGG pathway enrichment analysis of the target genes of upregulated and downregulated miRNAs was performed. The top 20 significantly enriched KEGG pathways are highlighted and summarized in Fig. 4. The genes that are targeted by miRNAs that were downregulated in the EOC group compared with the $\mathrm{NC}$ group were most significantly involved in pathways with cancer-promoting potential, such as phosphoinositide 3-kinase-Akt, Wnt or mitogen-activated protein kinase signaling pathways. These results suggested that changes in the expression of peritoneal exosomal miRNAs

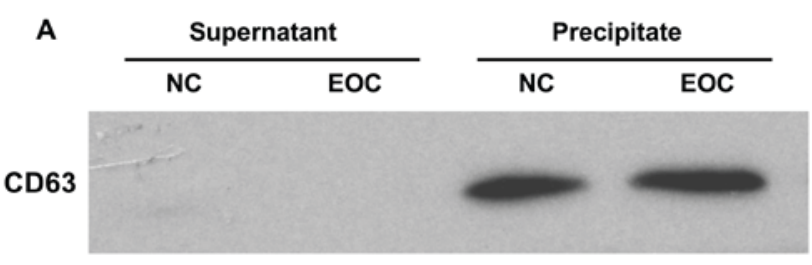

\section{B}

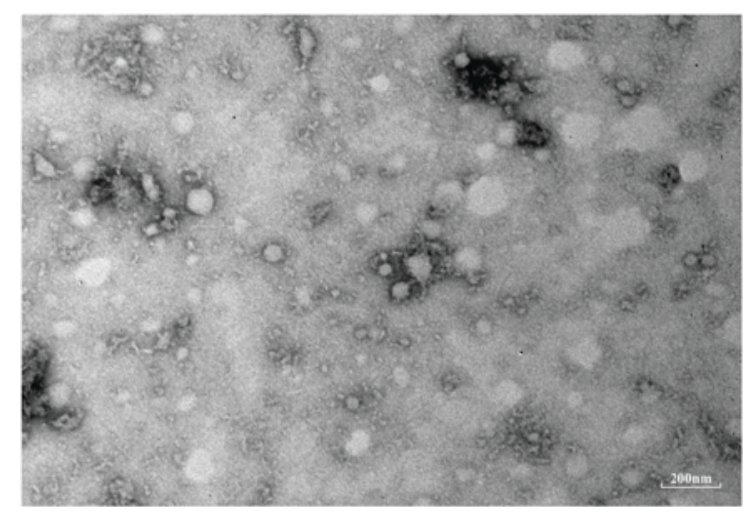

Figure 1. Verification of exosome precipitation. (A) Representative result of western blotting of precipitated exosomes and supernatant following precipitation for CD63. (B) Representative result of transmission electron microscopy of exosomes precipitated from samples of ascites from patients with epithelial ovarian cancer. NC, samples from participants without cancer; EOC, samples from patients with epithelial ovarian cancer.

might reflect and have potential effects on the physiopathological features of ovarian cancer.

Upregulation of miR-149-3p and miR-222-5p is associated with the progression of ovarian cancer. To further investigate the effect of changes in the expression of peritoneal exosomal miRNAs on the pathophysiology of ovarian cancer, the top 10 most significantly upregulated miRNAs with $\mathrm{TPM}_{\mathrm{EOC}}>50$ were sorted (Table IV). A total of three miRNAs with an increased expression that was associated with a decreased survival in patients with ovarian cancer were identified. As indicated in Fig. 5, a high expression of miR-149-3p and miR-222-5p was significantly correlated with a decrease in the 5-year and overall survival of patients with EOC as revealed by Kaplan-Meier curve analysis. A high expression of miR-1246 was also associated with a poor survival in patients with EOC, but there was no statistical significance $(\mathrm{P}>0.05)$. To the best of our knowledge, the effects of miR-149-3p and miR-222-5p on the pathophysiology of EOC have not been adequately characterized by other studies. Considering that these two miRNAs were significantly upregulated in EOC samples compared with the NC samples, it was inferred from these results that miR-149-3p and miR-222-5p may participate in the oncogenesis or progression of EOC.

The association between EOC and the upregulation of miR-149-3p or miR-222-5p in peritoneal exosomes was further verified by evaluating the expression level of these two miRNAs in the peritoneal exosomes that were obtained from the 10 patients with EOC and $10 \mathrm{NC}$ participants. As indicated in Fig. 6, RT-qPCR results indicated that the expression level of miR-149-3p and miR-222-5p was significantly increased in EOC samples compared with the NC samples. These data 


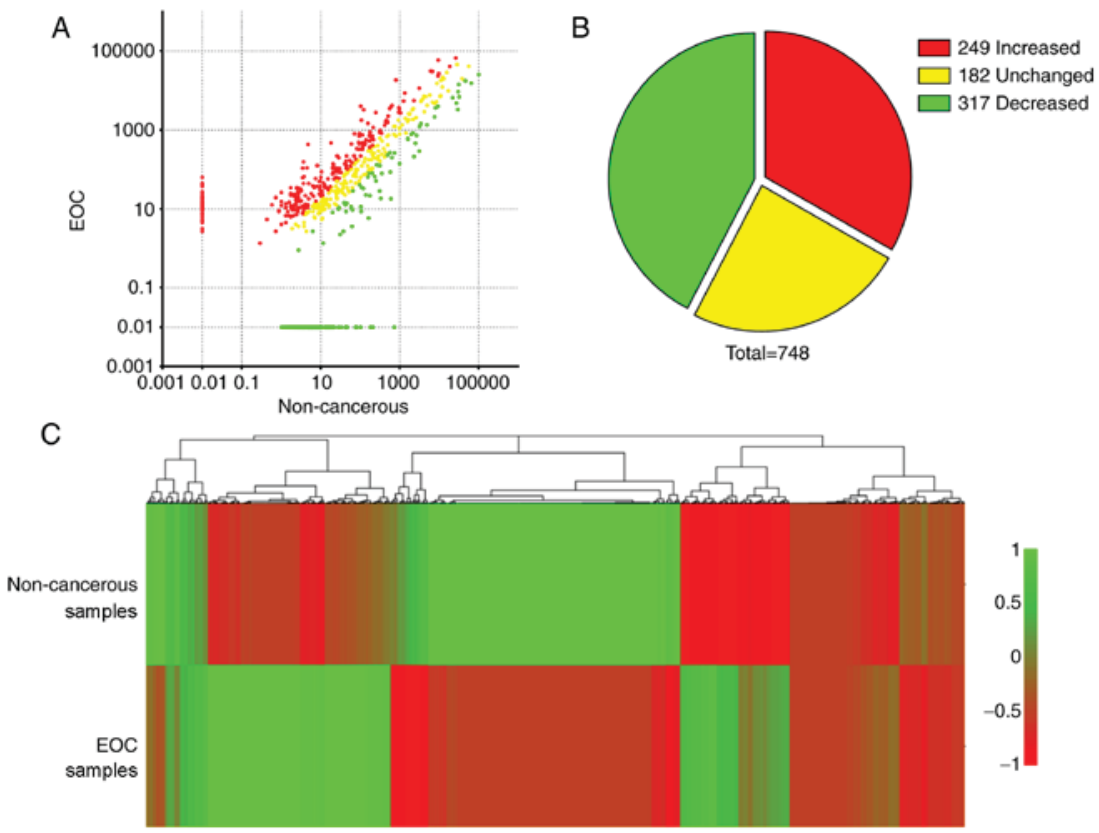

Figure 2. Comparison of microRNA expression between exosomes obtained from samples of ascites from patients with epithelial ovarian cancer and peritoneal lavage samples from participants without cancer. (A) Differentially expressed microRNAs as revealed by a scatter plot. Each dot represents an individual microRNA detected by high-throughput sequencing. Horizontal and vertical coordinates of each dot represent its $\log _{10}$ (TPM) in two sequencing results. (B) Pie chart summarizing the differential expression of all detected microRNAs. (C) Heat map summarizing the differential expression of all detected microRNAs. EOC, exosome sample from epithelial ovarian cancer patient. Red, upregulated microRNAs; green, downregulated microRNAs. EOC, samples from patients with epithelial ovarian cancer; TPM, transcripts per million.

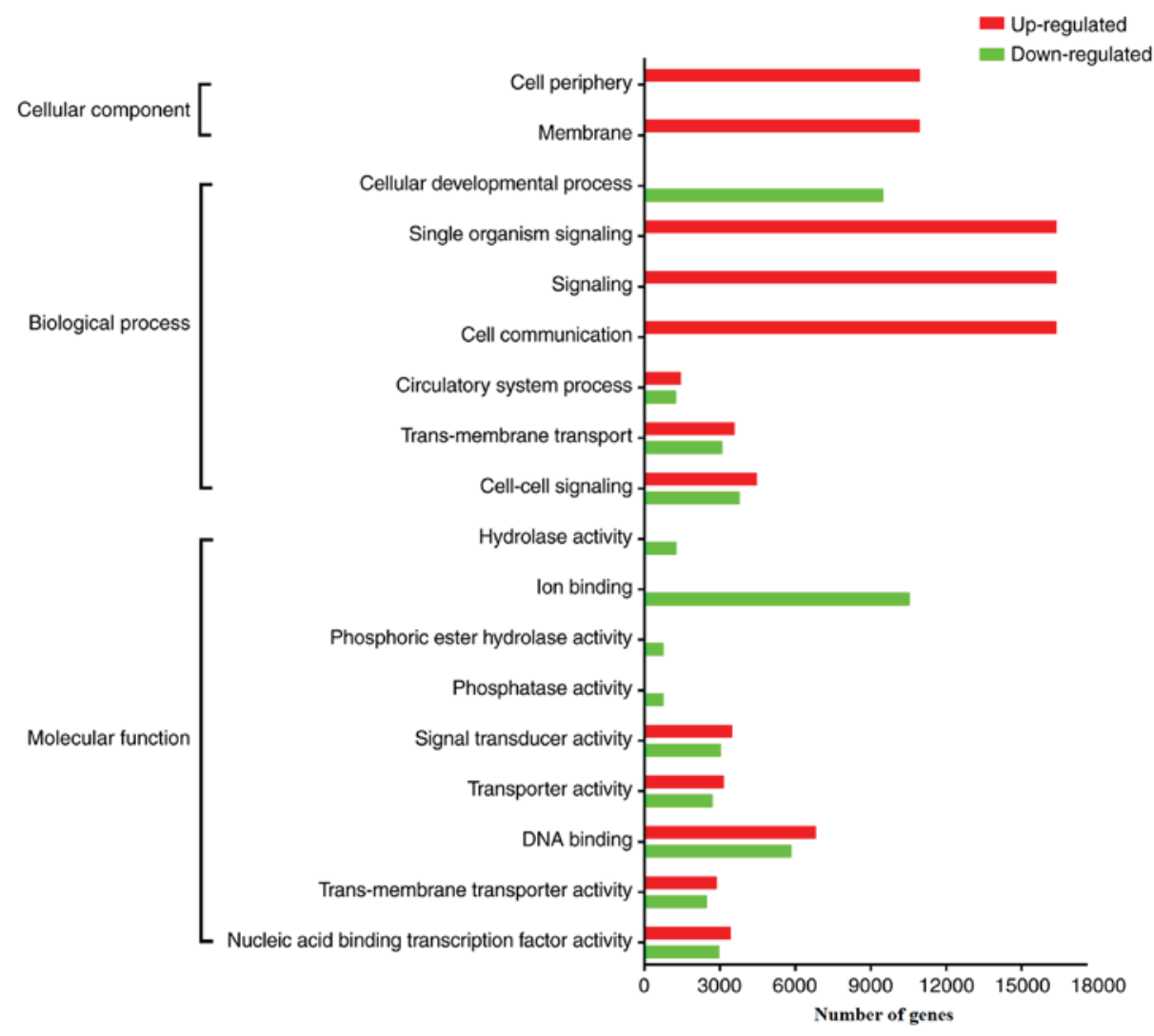

Figure 3. Gene Ontology enrichment annotation of predicted target genes of upregulated and downregulated microRNAs in exosome samples from patients with epithelial ovarian cancer compared with participants without cancer. Red, upregulated microRNAs; green, downregulated microRNAs.

suggested that miR-149-3p and miR-222-5p were associated with EOC and the miRNAs might be involved in the progression of EOC with the potential to serve as a biomarker for estimating the prognosis of patients with EOC. 
Table III. GO enrichment of the target genes of upregulated miRNAs $(\mathrm{P}<0.05)$.

Gene ratio: 44955 (total number of

GO ID

Description

differential genes) $(\%)$

P-value

Cellular component
GO:0005886
GO:0016020
GO:0071944

Molecular function

GO:0001071

GO:0022857

GO:0003677

GO:0005215

GO:0004871

Biological process

$\begin{array}{ll}\text { GO:0007267 } & \text { Cell-cell signaling } \\ \text { GO:0055085 } & \text { Trans-membrane transport } \\ \text { GO:0003013 } & \text { Circulatory system process } \\ \text { GO:0007154 } & \text { Cell communication } \\ \text { GO:0023052 } & \text { Signaling } \\ \text { GO:0044700 } & \text { Single organism signaling }\end{array}$

Plasma membrane

Membrane

Cell periphery

Nucleic acid binding transcription factor activity

Trans-membrane transporter activity

DNA binding

Transporter activity

Signal transducer activity

Single organism signaling
10947 (27.42)

10947 (27.42)

10955 (27.44)

$3440(7.65)$

2887 (6.42)

6824 (15.18)

3149 (7)

3485 (7.75)

4472 (10.12)

3575 (8.09)

1446 (3.27)

16385 (37.06)

16385 (37.06)

16385 (37.06)
0.018589

0.018589

0.018807

$\mathrm{P}<0.1$

0.000001

0.000001

0.00003

0.034531

$\mathrm{P}<0.1$

0.000132

0.000211

0.018231

0.018231

0.018231

GO, Gene Ontology.

Table IV. Top 10-upregulated miRNA with the highest fold change and TPM >50 in EOC exosomes.

\begin{tabular}{lccrrrrr}
\hline MicroRNA & Total reads in NC & Total reads in EOC & NC count & EOC count & TPM $_{\text {NC }}$ & TPM $_{\text {EOC }}$ & $\log _{2}(\mathrm{FC})$ \\
\hline hsa-miR-149-3p & 6893108 & 2217219 & 0 & 140 & 0.0100 & 63.1422 & 12.62438881 \\
hsa-miR-150-3p & 6893108 & 2217219 & 33 & 958 & 4.7874 & 432.0728 & 6.49588825 \\
hsa-miR-1246 & 6893108 & 2217219 & 25 & 345 & 3.6268 & 155.6003 & 5.42300384 \\
hsa-miR-1228-5p & 6893108 & 2217219 & 24 & 240 & 3.4817 & 108.2437 & 4.95834735 \\
hsa-miR-194-5p & 6893108 & 2217219 & 845 & 7238 & 122.5862 & 3264.4497 & 4.73497133 \\
hsa-miR-215-5p & 6893108 & 2217219 & 125 & 1046 & 18.1341 & 471.7621 & 4.70128247 \\
hsa-miR-671-3p & 6893108 & 2217219 & 31 & 244 & 4.4972 & 110.0478 & 4.61295945 \\
hsa-miR-192-5p & 6893108 & 2217219 & 5508 & 41272 & 799.0590 & 18614.3092 & 4.54196624 \\
hsa-miR-1197 & 6893108 & 2217219 & 24 & 138 & 3.4817 & 62.2401 & 4.15998057 \\
hsa-miR-222-5p & 6893108 & 2217219 & 26 & 147 & 3.7719 & 66.2993 & 4.13563031
\end{tabular}

FC, fold change; miR, microRNA; NC, participants without cancer; EOC, epithelial ovarian cancer; TPM, transcripts per million reads.

\section{Discussion}

The aim and scope of the present research was to compare the peritoneal exosomal miRNA profile between patients with ovarian cancer and participants without ovarian cancer in order to identify differentially expressed miRNAs that are potentially associated with peritoneal metastasis of ovarian cancer with considerable prognostic value. A recent research by Azais et al (21) reported that peritoneal metastases were diagnosed in $70-75 \%$ of all ovarian cancer cases. Abdominal cavity is a preferential metastatic site for ovarian cancer, and peritoneal exosomes or other extracellular vesicles have been characterized as important mediators of metastasis or development of ovarian cancer by transporting substances including proteins, mRNAs and miRNAs in an intercellular fashion and affecting the biological features of recipient cells $(18,22,23)$.

Of note, two major types of exosomes are supposed to be in the tumor microenvironment and of great relevance to tumor development, including tumor cell-derived exosomes and exosomes derived from non-tumoral cells (24). It is not possible to distinguish these two types of exosomes by using body fluids, including blood or ascites as specimens (25). The contents loaded in these exosomes may therefore be affected by different factors, including cellular origin or physiopathological condition, yielding different analysis results. The 
A

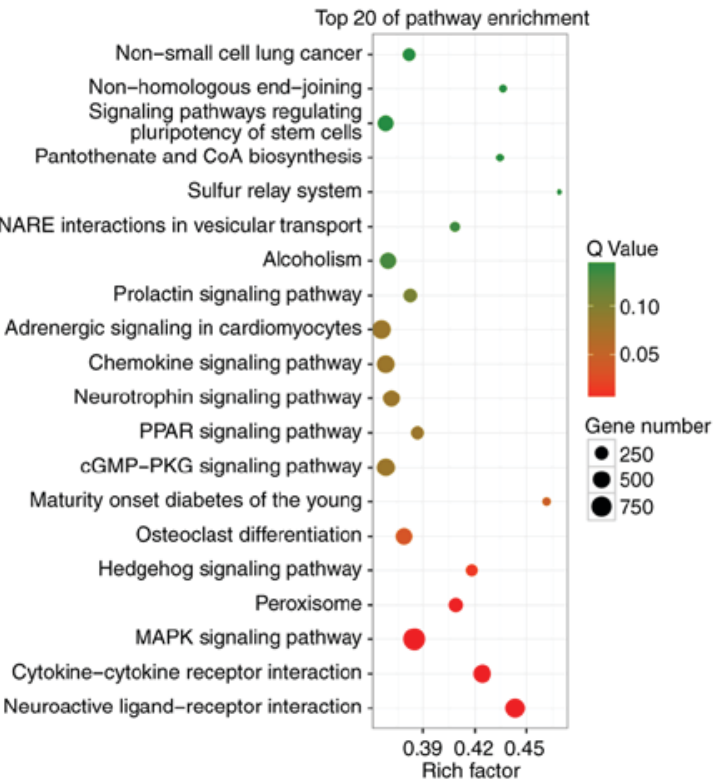

B

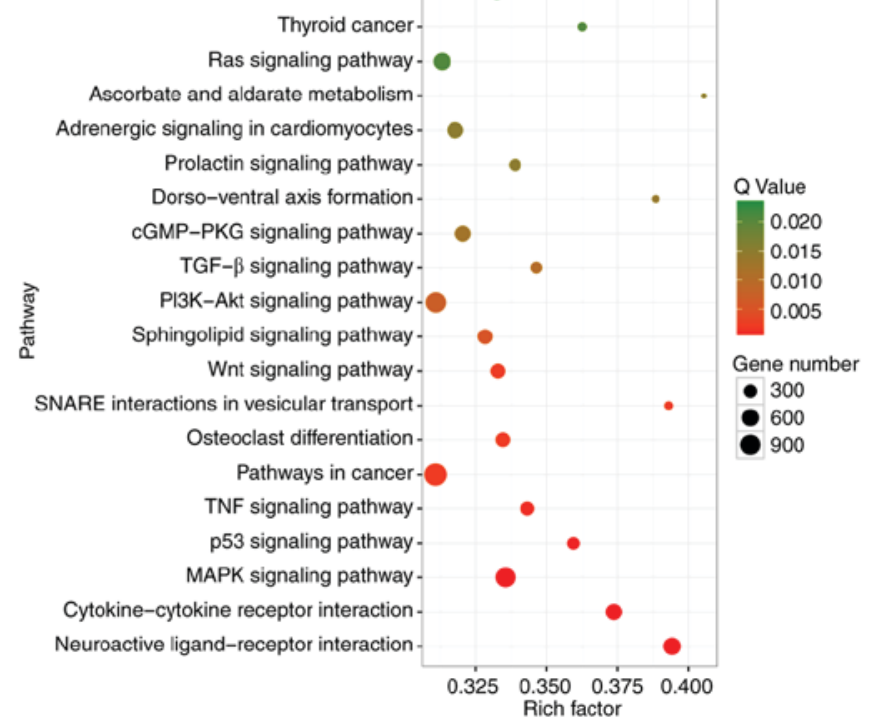

Figure 4. Kyoto Encyclopedia of Genes and Genomes pathway enrichment annotation of predicted target genes of upregulated and downregulated microRNAs in exosome samples from patients with epithelial ovarian cancer compared with participants without cancer. (A) Enrichment annotation of predicted target genes of upregulated microRNAs. (B) Enrichment annotation of predicted target genes of downregulated microRNAs. MAPK, mitogen-activated protein kinase; PI3K, phosphoinositide 3-kinase; TNF, tumor necrosis factor.
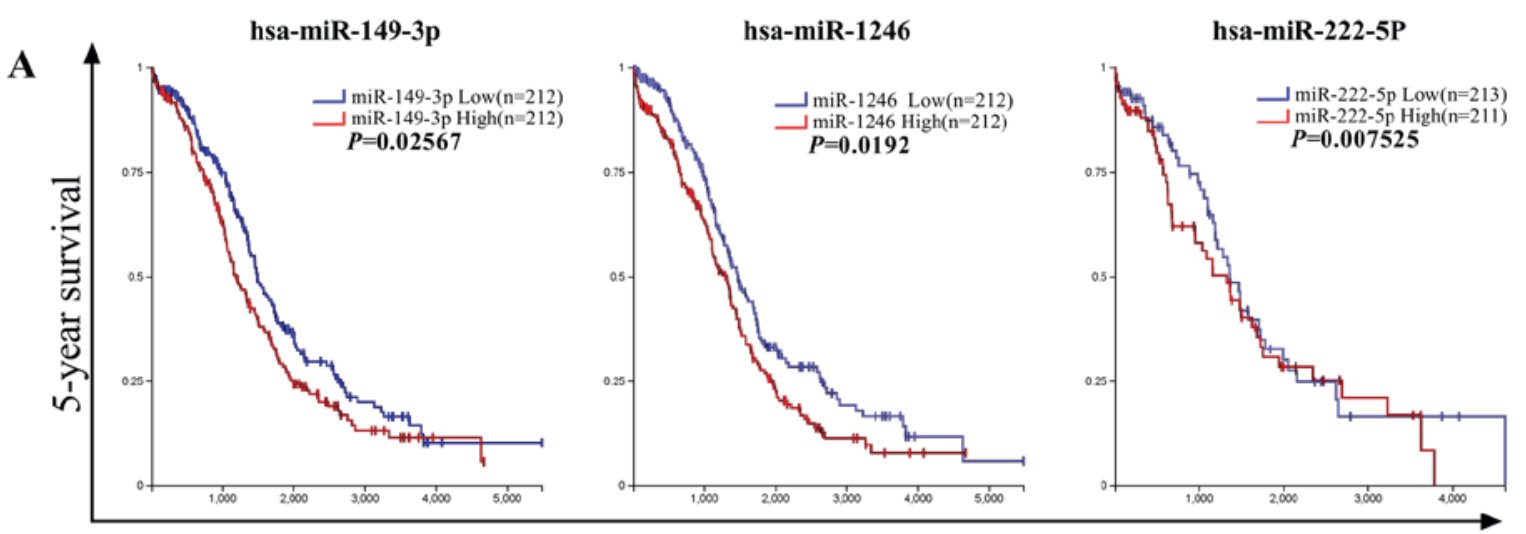

Time(day)
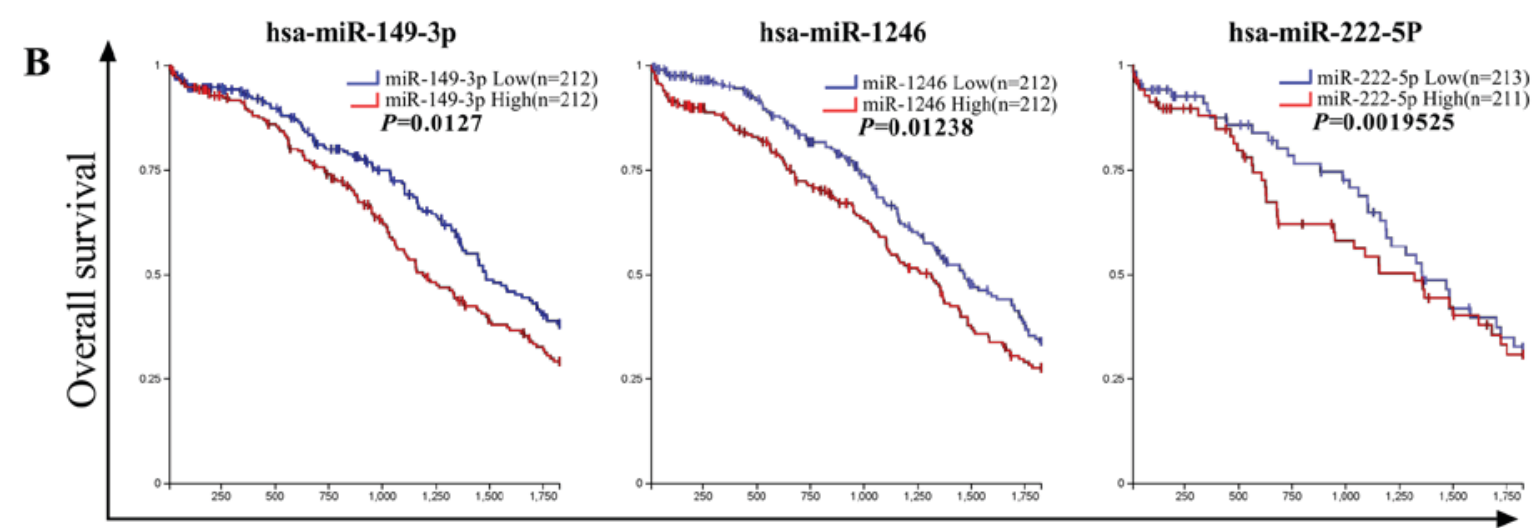

Time(day)

Figure 5. Kaplan-Meier curve analysis of the effects of miR-149-3p, miR-1246 and miR-222-5p expression levels on the (A) 5-year and (B) overall survival of patients with ovarian cancer as revealed by The Cancer Genome Atlas public data. miR, microRNA.

sequencing result in the present study indicated an upregulation of miR-149-3p and miR-222-5p in peritoneal exosomes that were acquired from patients with ovarian cancer compared with that from participants without cancer. The upregulation 

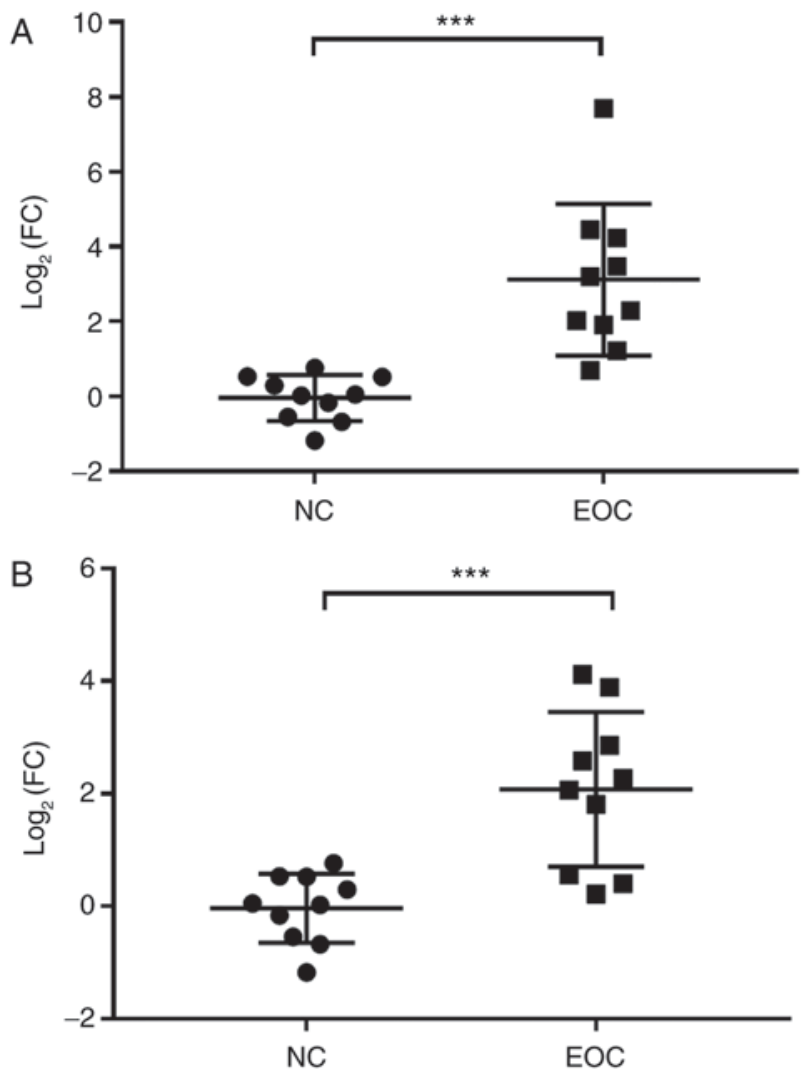

Figure 6. Comparison of (A) miR-149-3p and (B) miR-222-5p expression levels in participants without cancer and patients with epithelial ovarian cancer. The data are presented as the mean \pm standard deviation. ${ }^{* * *} \mathrm{P}<0.001$ EOC, samples from patients with epithelial ovarian cancer; FC, fold change of miRNA expression level in each sample comparing with the mean expression level in NC group; miR, microRNA; NC, samples from participants without cancer.

of miR-149-3p and miR-222-5p was significantly associated with ovarian cancer and poor patient survival, thereby strongly indicating their prognostic and therapeutic values. There appears to be no previous reports that link these two miRNAs to ovarian cancer progression. Vaksman et al (26) reported in 2011 that miR-222 was upregulated in the pleural effusion of patient with ovarian cancer; therefore miR-222 may aid the survival of ovarian cancer cells by targeting P21 (RAC1) activated kinase 1 and phosphatase and tensin homolog. However, the present study failed to determine whether the miR-222 described in the report by Vaksman et al (26) was miR-222-5p or miR-222-3p as no sequence related to this miRNA was provided in their report. Interestingly, a number of studies have proposed that miR-149-3p may act as a tumor suppressor in gastric, pancreatic and renal cell cancer (27-29). Considering the significant association between miR-149-3p expression and poor prognosis in patients with ovarian cancer revealed in the present study, these contradicting results may be a reflection of the heterogeneity in cancer types with different tissue origins.

To examine the potential effect of the upregulation of miR-149-3p and miR-483-5p in the development of ovarian cancer, using the DIANA-miRPath online software performs GO and KEGG enrichment analysis of genes in TarBase that have been validated as targets of given miRNAs. The result suggested that the target genes of miR-149-3p and miR-222-5p were significantly enriched in 19 pathways, including the antigen processing and presentation pathway, where it was identified that the two miRNAs target major histocompatibility complex class I (MHC I) gene (data not shown). Considering the GO enrichment analysis of predicted target genes of all upregulated miRNAs and the survival analysis in the present study, it was hypothesized that the upregulation of miR-149-3p and miR-222-5p might downregulate MHC I expression, impairing antigen presentation and therefore reducing the susceptibility of ovarian cancer cells to $\mathrm{T}$ cell cytotoxicity. This potential mechanism will be further investigated in our future research.

Collectively, the present study identified significant differences in peritoneal exosomal miRNA components between patients with ovarian cancer with peritoneal metastasis and participants without cancer, and it suggested the prognostic value of two peritoneal exosomal miRNAs, namely miR-149-3p and miR-222-5p. However, the relatively low number of samples might undermine these results. To further validate these results, a large-scale screening in patients with ovarian cancer is required in the future.

\section{Acknowledgements}

Not applicable.

\section{Funding}

The present study is supported by the 'Henan Provincial Higher Education Key Research Project Guidance Program', project number: 19B320022.

\section{Availability of data and materials}

All data used in this study are included in this published article.

\section{Authors' contributions}

YKL wrote the manuscript. YKL and CHL completed the experiments including extraction of extraperitoneal exosomes, exosomes identification and western blot. YML and WLW performed the experiments including extraction of exosomal miRNAs, construction of sequencing libraries and pre-processing of raw data. $\mathrm{BH}$ and $\mathrm{XQL}$ participated in analysis of miRNA expression, gene set enrichment analysis of the target genes of miRNAs. JQC participated in RT-qPCR assay, Kaplan-Meier curve analysis and statistical analysis. YKL and JQC revised the manuscript. All authors read and approved the final the manuscript.

\section{Ethics approval and consent to participate}

The present study was approved by the Medical Ethics Committee of the Second Affiliated Hospital of Zhengzhou University (Henan, China). Written informed consent was provided from each patient.

\section{Patient consent for publication}

Patients provided consent for the publication of this data and any associated images. 


\section{Competing interests}

The authors declare that they have no competing interests.

\section{References}

1. Jayson GC, Kohn EC, Kitchener HC and Ledermann JA: Ovarian cancer. Lancet 384: 1376-1388, 2014.

2. Thibault B, Castells M, Delord JP and Couderc B: Ovarian cancer microenvironment: Implications for cancer dissemination and chemoresistance acquisition. Cancer Metastasis Rev 33: $17-39,2014$

3. Nieman KM, Kenny HA, Penicka CV, Ladanyi A, Buell-Gutbrod R, Zillhardt MR, Romero IL, Carey MS, Mills GB, Hotamisligil GS, et al: Adipocytes promote ovarian cancer metastasis and provide energy for rapid tumor growth. Nat Med 17: 1498-1503, 2011.

4. Bapat SA, Mali AM, Koppikar CB and Kurrey NK: Stem and progenitor-like cells contribute to the aggressive behavior of human epithelial ovarian cancer. Cancer Res 65: 3025-3029, 2005

5. Alvero AB, Chen R, Fu HH, Montagna M, Schwartz PE, Rutherford T, Silasi DA, Steffensen KD, Waldstrom M, Visintin I and Mor G: Molecular phenotyping of human ovarian cancer stem cells unravels the mechanisms for repair and chemoresistance. Cell Cycle 8: 158-166, 2009.

6. Kajiyama H, Shibata K, Terauchi M, Yamashita M, Ino K, Nawa A and Kikkawa F: Chemoresistance to paclitaxel induces epithelial-mesenchymal transition and enhances metastatic potential for epithelial ovarian carcinoma cells. Int J Oncol 31: 277-283, 2007.

7. Zack TI, Schumacher SE, Carter SL, Cherniack AD, Saksena G, Tabak B, Lawrence MS, Zhsng CZ, Wala J, Mermel CH, et al: Pan-cancer patterns of somatic copy number alteration. Nat Genet 45: 1134-1140, 2013.

8. Patch AM, Christie EL, Etemadmoghadam D, Garsed DW, George J, Fereday S, Nones K, Cowin P, Alsop K, Bailey PJ, et al: Whole-genome characterization of chemoresistant ovarian cancer. Nature 521: 489-494, 2015.

9. Malek JA, Mery E, Mahmoud YA, Al-Azwani EK, Roger L, Huang R, Jouve E, Lis R, Thiery JP, Querleu D and Rafii A: Copy number variation analysis of matched ovarian primary tumors and peritoneal metastasis. PLoS One 6: e28561, 2011.

10. Bracken CP, Scott HS and Goodall GJ: A network-biology perspective of microRNA function and dysfunction in cancer. Nat Rev Genet 17: 719-732, 2016.

11. Lin S and Gregory RI: MicroRNA biogenesis pathways in cancer. Nat Rev Cancer 15: 321-333, 2015.

12. O'Connell RM, Rao DS and Baltimore D: microRNA regulation of inflammatory responses. Annu Rev Immunol 30: 295-312, 2012.

13. Deb B, Uddin A and Chakraborty S: miRNAs and ovarian cancer: An overview. J Cell Physiol 233: 3846-3854, 2018.

14. Mihanfar A, Fattahi A and Nejabati HR: MicroRNA-mediated drug resistance in ovarian cancer. J Cell Physiol: Jun 19, 2017 (Epub ahead of print).
15. Palma Flores C, García-Vázquez R, Gallardo Rincón D, Ruiz-García E, Astudillo de la Vega H, Marchat LA, Salinas Vera YM and López-Camarillo C: MicroRNAs driving invasion and metastasis in ovarian cancer: Opportunities for translational medicine (Review). Int J Oncol 50: 1461-1476, 2017.

16. Raposo G and Stoorvogel W: Extracellular vesicles: Exosomes, microvesicles, and friends. J Cell Biol 200: 373-383, 2013.

17. Li $X$ and Wang $X$ : The emerging roles and therapeutic potential of exosomes in epithelial ovarian cancer. Mol Cancer 16: 92 , 2017.

18. Nakamura K, Sawada K, Kinose Y, Yoshimura A, Toda A, Nakatsuka E, Hashimoto K, Mabuchi S, Morishige KI, Kurachi H, et al: Exosomes promote ovarian cancer cell invasion through transfer of CD44 to peritoneal mesothelial cells. Mol Cancer Res 15: 78-92, 2017.

19. Tang MK and Wong AS: Exosomes: Emerging biomarkers and targets for ovarian cancer. Cancer Lett 367: 26-33, 2015.

20. Armstrong RN, Colyer HA and Mills KI: Screening for miRNA expression changes using quantitative PCR (Q-PCR). Methods Mol Biol 863: 293-302, 2012

21. Azaïs H, Estevez JP, Foucher P, Kerbage Y, Mordon S and Collinet P: Dealing with microscopic peritoneal metastases of epithelial ovarian cancer. A surgical challenge. Surg Oncol 26: 46-52, 2017

22. Yokoi A, Yoshioka Y, Yamamoto Y, Ishikawa M, Ikeda SI, Kato T, Kiyono T, Takeshita F, Kajiyama H, Kikkawa F and Ochiya T: Malignant extracellular vesicles carrying MMP1 mRNA facilitate peritoneal dissemination in ovarian cancer. Nature Commun 8: 14470, 2017.

23. Keller S, König AK, Marmé F, Runz S, Wolterink S, Koensgen D, Mustea A, Sehouli J and Altevogt P: Systemic presence and tumor-growth promoting effect of ovarian carcinoma released exosomes. Cancer Lett 278: 73-81, 2009.

24. Wang Z, Chen JQ, Liu JL and Tian L: Exosomes in tumor microenvironment: Novel transporters and biomarkers. J Transl Med 14: 297, 2016.

25. Kumar D, Gupta D, Shankar S and Srivastava RK: Biomolecular characterization of exosomes released from cancer stem cells: Possible implications for biomarker and treatment of cancer. Oncotarget 6: 3280-3291, 2015.

26. Vaksman O, Stavnes HT, Kaern J, Trope CG, Davidson B and Reich R: miRNA profiling along tumour progression in ovarian carcinoma. J Cell Mol Med 15: 1593-1602, 2011.

27. Cao D, Jia Z, You L, Wu Y, Hou Z, Suo Y, Zhang H, Wen S, Tsukamoto T, Oshima M, et al: $18 \beta$-glycyrrhetinic acid suppresses gastric cancer by activation of miR-149-3p-Wnt-1 signaling. Oncotarget 7: 71960-71973, 2016.

28. Okato A, Arai T, Yamada Y, Sugawara S, Koshizuka K, Fujimura L, Kurozumi A, Kato M, Kojima S, Naya Y, et al: Dual strands of pre-miR-149 inhibit cancer cell migration and invasion through targeting FOXM1 in renal cell carcinoma. Int J Mol Sci 18: pii: E1969, 2017.

29. Si L, Xu L, Yin L, Qi Y, Han X, Xu Y, Zhao Y, Liu K and Peng J: Potent effects of dioscin against pancreatic cancer via miR-149-3P-mediated inhibition of the Akt1 signalling pathway. Br J Pharmacol 174: 553-568, 2017. 